\title{
GENERALIZED VOLTERRA COMPANION OPERATORS ON FOCK SPACES
}

\author{
TESFA MENGESTIE
}

\begin{abstract}
We characterize the bounded and compact generalized Volterra companion integral operators on Fock spaces acting between the standard Fock spaces. As a special case, we prove that there exist no nontrivial compact Volterra companion integral and multiplication operators on Fock spaces. We also obtain asymptotic estimates for the norm of these operators.
\end{abstract}

\section{INTRODUCTION}

For holomorphic functions $f$ and $g$, the Volterra type integral operator $V_{g}$ and its companion $J_{g}$ are defined by

$$
V_{g} f(z)=\int_{0}^{z} f(w) g^{\prime}(w) d w \text { and } J_{g} f(z)=\int_{0}^{z} f^{\prime}(w) g(w) d w .
$$

Applying integration by parts in any one of the above integrals gives the relation

$$
V_{g} f+J_{g} f=M_{g} f-f(0) g(0),
$$

where $M_{g} f=g f$ is the multiplication operator of symbol $g$. These integral type operators have been studied extensively on various spaces of analytic functions with the aim to explore the connection between their operator theoretic behaviours with the function theoretic properties of the symbols $g$ especially after the works of Pommerenke [16], and Aleman and Siskakis [3, 4] on Hardy and Bergman spaces. For more information on the subject, we refer to $[1,2,19]$ and the related references therein.

The idea to extend the operators $V_{g}$ and $J_{g}$ was first raised by $\mathrm{S}$. Li and S. Stević in 2006. They eventually proposed introducing the following operators induced by pairs of holomorphic symbols $(g, \psi)$ :

$$
\begin{array}{r}
V_{g}^{\psi} f(z)=\int_{0}^{z} f(\psi(w)) g^{\prime}(w) d w, \quad C_{g}^{\psi} f(z)=\int_{0}^{\psi(z)} f(w) g^{\prime}(w) d w \\
J_{(g, \psi)} f(z)=\int_{0}^{z} f^{\prime}(\psi(w)) g(w) d w, \text { and } C_{(g, \psi)} f(z)=\int_{0}^{\psi(z)} f^{\prime}(w) g(w) d w
\end{array}
$$

2000 Mathematics Subject Classification. 30E05, 46E22. 
and studied their operator theoretic properties in terms of the pairs $(g, \psi)$ on some spaces of analytic functions on the unit disk [11,12]. For more recent results on the class of operators in (1.1), one may consult the materials for instance in [14, 15, 22].

Studying operators $V_{g}^{\psi}$ and $C_{g}^{\psi}$ attracted somewhat more attention partly due to the fact that their bounded and compact properties are related to the notion of Carleson measures, which are readily available for several known spaces. In contrast, relatively little is known on the operators $J_{(g, \psi)}$ and $C_{(g, \psi)}$ apart from some cases where the target space is restricted to be a growth type space with norm defined in terms of derivatives. We may mention that these class of operators have also found applications in the study of linear isometries of spaces of analytic functions. A prototype example in this case is the following. Let $S^{p}$ denotes the space of all analytic functions $f$ in the unit disc for which its derivative $f^{\prime}$ belongs to the Hardy space $H^{p}$. Then it has been show that for $p \neq 2$, any surjective isometry $T$ of $S^{p}$ under the norm $\|f\|_{S^{p}}=|f(0)|+\left\|f^{\prime}\right\|_{H^{p}}$ is of the form

$$
T f=\lambda f(0)+\lambda J_{(g, \psi)} f
$$

for some unimodular $\lambda$ in $\mathbb{C}$, a nonconstant inner function $\psi$ and a function $g$ in $H^{p}$ [6].

The bounded and compact properties of the class of operators in (1.1) when acting between Fock spaces were studied in $[14,15]$. In this note, we continue that line of research for the remaining class of operators $J_{(g, \psi)}$ and $C_{(\psi, g)}$ which are called the generalized Volterra companion integral operators. Observe that the particular choice $\psi(z)=z$ reduces both $J_{(g, \psi)}$ and $C_{(\psi, g)}$ to the operator $J_{g}$. On the other hand, setting $g=\psi^{\prime}$ and $g=1$ respectively reduce the operators $J_{(g, \psi)}$ and $C_{(\psi, g)}$ to the composition operator $C_{\psi}$ up to a constant. As will be seen latter, a consequence of our main results shows that there exist no nontrivial compact Volterra companion integral and multiplication operators acting between Fock spaces.

The classical weighted Fock space $\mathcal{F}_{\alpha}^{p}$ consists of all entire functions $f$ for which

$$
\|f\|_{p}^{p}=\frac{\alpha p}{2 \pi} \int_{\mathbb{C}}|f(z)|^{p} e^{-\frac{\alpha p}{2}|z|^{2}} d m(z)<\infty
$$

where $0<p<\infty, \alpha>0$ is a parameter, and $d m$ denotes the usual Lebesgue area measure on $\mathbb{C}$. For $p=\infty$, the growth type space $\mathcal{F}_{\alpha}^{\infty}$ contains all entire functions $f$ such that

$$
\|f\|_{\infty}=\sup _{z \in \mathbb{C}}|f(z)| e^{-\frac{\alpha}{2}|z|^{2}}<\infty .
$$

The space $\mathcal{F}_{\alpha}^{2}$, in particular, is a reproducing kernel Hilbert space with kernel function $K_{w}(z)=e^{\alpha\langle z, w\rangle}$ and normalized kernel function $k_{w}(z)=e^{\alpha\langle z, w\rangle}-\alpha|w|^{2} / 2$.

We close this introduction with a word on notation: The notation $U(z) \lesssim V(z)$ (or equivalently $V(z) \gtrsim U(z)$ ) means that there is a constant $C$ such that $U(z) \leq C V(z)$ 
holds for all $z$ in the set of a question. We write $U(z) \simeq V(z)$ if both $U(z) \lesssim V(z)$ and $V(z) \lesssim U(z)$. In addition, we denote by $L^{p}$ the Lebesque spaces $L^{p}(\mathbb{C}, d m)$.

\section{THE MAIN RESULTS}

In this section, we will present our main results. We may first set

$$
P_{\psi}(z)=\frac{e^{\frac{\alpha}{2}\left(|\psi(z)|^{2}-|z|^{2}\right)}}{1+|z|} \quad \text { and } \quad Q_{g}(z)=\frac{|g(z)| e^{-\frac{\alpha}{2}|z|^{2}}}{1+|z|} .
$$

Then our results are expressed in terms of the functions

$M_{(g, \psi)}(z)=|g(z)|(|\psi(z)|+1) P_{\psi}(z) \quad$ and $\quad M_{(g(\psi), \psi)}(z)=|g(\psi(z))|(|\psi(z)|+1) P_{\psi}(z)$,

and generalized Berezin type integral transforms:

$$
B_{(|g(\psi)| q, \psi)}(w)=\int_{\mathbb{C}}\left|k_{w}(\psi(z))\right|^{q}\left((|w|+1)\left|\psi^{\prime}(z)\right| Q_{g(\psi)}(z)\right)^{q} d m(z)
$$

and

$$
B_{\left(|g|^{q}, \psi\right)}(w)=\int_{\mathbb{C}}\left|k_{w}(\psi(z))\right|^{q}\left((|w|+1) Q_{g}(z)\right)^{q} d m(z)
$$

We now state our first main result.

Theorem 2.1. Let $0<p \leq q \leq \infty$ and $(g, \psi)$ be a pair of entire functions. Then

(i) $J_{(g, \psi)}: \mathcal{F}_{\alpha}^{p} \rightarrow \mathcal{F}_{\alpha}^{q}$ is bounded if and only if $M_{(g, \psi)}$ when $q=\infty$ and $B_{\left(|g|^{q}, \psi\right)}$ when $q<\infty$ belong to $L^{\infty}$. In this case, we also have

$$
\left\|J_{(g, \psi)}\right\| \simeq \begin{cases}\left\|M_{(g, \psi)}\right\|_{L^{\infty}}, & q=\infty \\ \left\|B_{(|g| q, \psi}\right\|_{L^{\infty}}, & q<\infty .\end{cases}
$$

(ii) $J_{(g, \psi)}: \mathcal{F}_{\alpha}^{p} \rightarrow \mathcal{F}_{\alpha}^{q}$ is compact if and only if

(a) $\lim _{|z| \rightarrow \infty} B_{\left(|g|^{q}, \psi\right)}(z)=0$ for $q<\infty$;

(b) it is bounded and $\lim _{|\psi(z)| \rightarrow \infty} M_{(g, \psi)}(z)=0$ when $q=\infty$.

(iii) $C_{(g, \psi)}: \mathcal{F}_{\alpha}^{p} \rightarrow \mathcal{F}_{\alpha}^{q}$ is bounded if and only if $M_{(g(\psi), \psi)}$ when $q=\infty$ and $B_{\left(|g(\psi)|^{q}, \psi\right)}$ when $q<\infty$ belong to $L^{\infty}$. We also estimate the norm by

$$
\left\|C_{(g, \psi)}\right\| \simeq \begin{cases}\left\|M_{(g(\psi), \psi)}\right\|_{L^{\infty}}, & q=\infty \\ \left\|B_{\left(|g(\psi)|^{q}, \psi\right)}\right\|_{L^{\infty}}^{1 / q}, & q<\infty .\end{cases}
$$

(iv) $C_{(g, \psi)}: \mathcal{F}_{\alpha}^{p} \rightarrow \mathcal{F}_{\alpha}^{q}$ is compact if and only if
(a) $\lim _{|z| \rightarrow \infty} B_{\left(|g(\psi)|^{q}, \psi\right)}(z)=0$ for $q<\infty$;
(b) it is bounded and $\lim _{|\psi(z)| \rightarrow \infty} M_{(g(\psi), \psi)}(z)=0$ when $q=\infty$. 
It may be noted that the conditions on the preceding results do not depend on exponent $p$ from the domain space $\mathcal{F}_{\alpha}^{p}$ apart from the sole assumption that $p \leq q$, where $q$ is the exponent on the target space. It means that if there exists a $p_{o} \leq q$ for which the map $J_{(g, \psi)}$ or $C_{(g, \psi)}$ is bounded (compact) from $\mathcal{F}_{\alpha}^{p_{o}}$ to $\mathcal{F}_{\alpha}^{q}$, the same conclusion holds when we replace the domain space by $\mathcal{F}_{\alpha}^{p}$ for any $p \leq q$. A similar phenomena was observed in $[14,15]$ with the class of operators in $(1.1)$.

Recall that since the Fock spaces are nested, $\mathcal{F}_{\alpha}^{p} \subseteq \mathcal{F}_{\alpha}^{p}$ whenever $p \leq q$ [9], the conditions in Theorem 2.1 are from mapping smaller spaces into larger spaces under the maps $J_{(g, \psi)}$ and $C_{(g, \psi)}$. Conversely, when we map larger spaces into smaller spaces with the same mappings, we get the following stronger integrability conditions.

Theorem 2.2. Let $0<q<p \leq \infty$ and $(g, \psi)$ be a pair of entire functions. Then

(i) $J_{(g, \psi)}: \mathcal{F}_{\alpha}^{p} \rightarrow \mathcal{F}_{\alpha}^{q}$ is bounded (compact) if and only if $B_{\left(|g|^{q}, \psi\right)}$ belongs to $L^{1}$ when $p=\infty$ and to $L^{p /(p-q)}$ whenever $p<\infty$. Furthermore, we have

$$
\left\|J_{(g, \psi)}\right\| \simeq \begin{cases}\left\|B_{\left(|g|^{q}, \psi\right)}\right\|_{L^{1}}, & p=\infty \\ \left\|B_{\left(|g|^{q}, \psi\right)}\right\|_{L^{\frac{p}{p-q}}}, & p<\infty .\end{cases}
$$

(ii) $C_{(g, \psi)}: \mathcal{F}_{\alpha}^{p} \rightarrow \mathcal{F}_{\alpha}^{q}$ is bounded (compact) if and only if $B_{\left(|g \psi|^{q}, \psi\right)}$ belongs to $L^{1}$ for $p=\infty$ and to $L^{p /(p-q)}$ whenever $p<\infty$. Furthermore, we have

$$
\left\|J_{(g, \psi)}\right\| \simeq \begin{cases}\left\|B_{\left(|g(\psi)|^{q}, \psi\right)}\right\|_{L^{1}}, & p=\infty \\ \left\|B_{\left(|g(\psi)|^{q}, \psi\right)}\right\|_{L^{\frac{p}{p-q}}}, & p<\infty .\end{cases}
$$

Unlike the case for Theorem 2.1, the conditions in the preceding theorem rely on both the domain and the targets space exponents $p$ and $q$ except for the case when $p=\infty$. In this case, the corresponding condition is independent of the target space exponent $q$ as long as $q<p$.

From the relation $V_{g} f+J_{g} f=M_{g} f-f(0) g(0)$, we observe that if any two of the operators $V_{g}, J_{g}$ and $M_{g}$ are bounded so does the third onve. Interestingly, in Fock spaces more can be said, namely that $M_{g}$ is bounded (compact) if and only if so is the operator $J_{g}$. We formulate this observation as a corollary below and prove our assertion in the next section.

Corollary 2.1. Let $g$ be an entire function on $\mathbb{C}$. Then

(i) if $0<p \leq q \leq \infty$, then the operator $J_{g}: \mathcal{F}_{\alpha}^{p} \rightarrow \mathcal{F}_{\alpha}^{q}$ is bounded (respect. compact) if and only if so is $M_{g}: \mathcal{F}_{\alpha}^{p} \rightarrow \mathcal{F}_{\alpha}^{q}$, and this holds if and only if $g$ is a constant (respect. zero) function.

(ii) if $0<q<p \leq \infty$, then the operator $J_{g}: \mathcal{F}_{\alpha}^{p} \rightarrow \mathcal{F}_{\alpha}^{q}$ is bounded or compact if and only if so is $M_{g}: \mathcal{F}_{\alpha}^{p} \rightarrow \mathcal{F}_{\alpha}^{q}$, and this holds if and only if $g$ is the zero function. 
The results in the corollary verify that there exist no nonzero compact Volterra companion integral and multiplication operators acting between Fock spaces. Furthermore, it has now become clear that more symbols $g$ are admissible in inducing bounded or compact $V_{g}$ than $J_{g}$. More specifically, a number of results from $[5,14,15]$ ensure that $V_{g}$ : $\mathcal{F}_{\alpha}^{p} \rightarrow \mathcal{F}_{\alpha}^{q}, 0<p \leq q \leq \infty$, is bounded if and only if $g$ is a complex polynomial of degree not exceeding 2 , and its compactness holds if and only if its degree does not exceed 1 . On the other hand, if $p>q$, then $V_{g}$ is bounded or compact if and only if $g$ is again a polynomial of degree not exceeding 1 . It means that the bounded (compact) properties of the sum $V_{g}+J_{g}$ merely depends on the boundedness (compactness) of the summand $J_{g}$ when all the three operators act between Fock spaces.

By simply scaling $z$ as $\beta z$ for some $|\beta|<1$, it is easily seen that a wider class of symbols $g$ are admissible in giving rise to bounded (compact) $J_{(g, \psi)}$ than those $g$ guaranteed in the corollary. For instance if we set $\psi(z)=\beta|s|,|\beta|<1$ and $g$ be entire function such that $|g(z)|^{2} \leq e^{\gamma|z|^{2}}$ where $|\beta|^{2}+\gamma<1$. Then the pair $(g, \psi)$ satisfies the condition in part (i) of Theorem 2.1. This gives another impetus for the need to take further the study of Volterra companion integral and composition operators to the generalized cases $J_{(g, \psi)}$ and $C_{(\psi, g)}$.

Combining the results from Theorem 2.1, Theorem 2.2 and the corresponding boundedness (compactness) results from $[14,15]$, we immediately deduce the following.

Corollary 2.2. Let $g$ be a holomorphic function on $\mathbb{C}$ and $0<p, q \leq \infty$. Then if

(i) $J_{(g, \psi)}: \mathcal{F}_{\alpha}^{p} \rightarrow \mathcal{F}_{\alpha}^{q}$ is bounded (compact) so is the map $V_{g}^{\psi}: \mathcal{F}_{\alpha}^{p} \rightarrow \mathcal{F}_{\alpha}^{q}$.

(ii) $C_{(g, \psi)}: \mathcal{F}_{\alpha}^{p} \rightarrow \mathcal{F}_{\alpha}^{q}$ is bounded (compact) so is the map $C_{g}^{\psi}: \mathcal{F}_{\alpha}^{p} \rightarrow \mathcal{F}_{\alpha}^{q}$.

The converses of the statements in the corollary in general fail. To find a simple counterexample, we may simply set $\psi(z)=z$. Then the class of operators in (1.1) reduces to the Volterra type integral operator $V_{g}$ while those at (1.2) reduce to its companion operator $J_{g}$. Then the desired conclusion follows from the analysis in the paragraph immediately after Corollary 2.1 above.

\section{Auxiliary Results}

In this section we collect some auxiliary results that will be used in our subsequent considerations. Our first lemma provides a criteria for compactness of $J_{(g, \psi)}$ and $C_{(g, \psi)}$ when acting between Fock spaces.

Lemma 3.1. Let $0<q, p \leq \infty$ and $(g, \psi)$ be a pair of entire functions. Then

(i) $J_{(g, \psi)}: \mathcal{F}_{\alpha}^{p} \rightarrow \mathcal{F}_{\alpha}^{q}$ is compact if and only if $\left\|J_{(g, \psi)} f_{n}\right\|_{q} \rightarrow 0$ as $n \rightarrow \infty$ for each uniformly bounded sequence $\left(f_{n}\right)_{n \in \mathbb{N}}$ in $\mathcal{F}_{\alpha}^{p}$ converging to zero uniformly on compact subsets of $\mathbb{C}$ as $n \rightarrow \infty$. 
(ii) A similar statement holds when we replace the operator $J_{(g, \psi)}$ by $C_{(g, \psi)}$ in $(i)$.

The lemma can be proved following standard arguments, and will be used repeatedly in what follows without mentioning it over and over again.

For $q \geq 0$, we set $\varphi_{q}(z)=(1+|z|)^{q}$ to be a weight function on $\mathbb{C}$. Then for each $p \geq 1$, we introduce weighted $L_{\varphi_{q}}^{p}$ spaces consisting of all measurable functions $f$ on $\mathbb{C}$ such that

$$
\|f\|_{L_{\varphi_{q}}^{p}}^{p}=\int_{\mathbb{C}}\left|f(z) \varphi_{q}(z)\right|^{p} d m(z)<\infty
$$

for finite $p$ and when $p=\infty$, the corresponding norm is given by

$$
\|f\|_{L_{\varphi_{q}}^{\infty}}=\sup _{z \in \mathbb{C}}\left|f(z) \varphi_{q}(z)\right|<\infty .
$$

For a Borel measure $\mu$ on $\mathbb{C}$, we also define a Berezin type integral transform associated with it by

$$
\widetilde{\mu}_{q}(z)=\int_{\mathbb{C}}(1+|z|)^{q}\left|k_{z}(\zeta)\right|^{q} e^{-\frac{\alpha q}{2}|\zeta|^{2}} d \mu(\zeta) .
$$

In particular when $\mu$ is a measure such that $d \mu(z)=f(z) d m(z)$ for a given measurable function $f$, we prove the following.

Lemma 3.2. let $1 \leq p \leq \infty, r>0$ and $0 \leq q<\infty$. Then the operators $f \mapsto f_{r}$ and $f \mapsto \widetilde{f}_{q}$ from $L_{\varphi_{q}}^{p}$ to $L^{p}$ are bounded where $f_{r}(z)=(1+|z|)^{q} \mu(D(z, r)), D(z, r)=$ $\{\zeta \in \mathbb{C}:|z-\zeta|<r\}$, and

$$
\widetilde{f}_{q}(z)=\int_{\mathbb{C}}(1+|z|)^{q}\left|k_{z}(\zeta)\right|^{q} e^{-\frac{\alpha q}{2}|\zeta|^{2}} d \mu(\zeta)
$$

Proof. We mention that for the case when $q=0$, the lemma was first proved in [7]. We use interpolation arguments between the Lebesgue spaces $L_{w}^{p}$ and $L^{p}$ and extend the techniques there. Thus, it suffices to establish the statements for $p=1$ and $p=\infty$. We begin with the case $p=1$ and apply Fubini's theorem to estimate

$$
\begin{aligned}
\left\|\tilde{f}_{q}\right\|_{L^{1}} & =\int_{\mathbb{C}}\left|\int_{\mathbb{C}}\right|(1+|z|)^{q}\left|k_{z}(w)\right|^{q} e^{-\frac{\alpha q}{2}|w|^{2}} d \mu(w) \mid d m(z) \\
& \leq \int_{\mathbb{C}}\left(\int_{\mathbb{C}}\left|k_{z}(w)\right|^{q} e^{-\frac{\alpha q}{2}|w|^{2}}(1+|z|)^{q} d m(z)\right)|f(w)| d m(w) \\
& =\int_{\mathbb{C}}\left(\int_{\mathbb{C}}(1+|z|)^{q} e^{-\frac{\alpha q}{2}|z-w|^{2}} d m(z)\right)|f(w)| d m(w) \\
& \lesssim \int_{\mathbb{C}}(1+|w|)^{q}|f(w)| d m(w)=\|f\|_{L_{\varphi_{q}}^{1}} .
\end{aligned}
$$


On the other hand, applying Fubini's theorem again and the fact that $\chi_{D(\zeta, r)}(z)=$ $\chi_{D(z, r)}(\zeta)$ for all $\zeta$ and $z$ in $\mathbb{C}$, we have

$$
\begin{aligned}
\left\|f_{r}\right\|_{L^{1}}=\int_{\mathbb{C}}(1+|z|)^{q} \mu(D(z, r)) d m(z) & \leq \int_{\mathbb{C}}(1+|z|)^{q} \int_{D(z, r)}|f(\zeta)| d m(\zeta) d m(z) \\
=\int_{\mathbb{C}}|f(\zeta)| \int_{\mathbb{C}} \chi_{D(\zeta, r)}(z)(1+|z|)^{q} d m(z) d m(\zeta) & \simeq \int_{\mathbb{C}}|f(\zeta)|(1+|\zeta|)^{q} d m(\zeta)=\|f\|_{L_{\varphi_{q}}^{1}},
\end{aligned}
$$

where the last estimate follows since $1+|z| \simeq 1+|\zeta|$ for each $z$ in $D(\zeta, r)$.

We now proceed to show the case for $p=\infty$. For each $f \in L_{\varphi_{q}}^{\infty}$, it easily follows that

$$
\begin{array}{rl}
\sup _{z \in \mathbb{C}}\left|f_{r}(z)\right| \leq \sup _{z \in \mathbb{C}}(1+|z|)^{q} \int_{D(z, r)}|f(\zeta)| d & m(\zeta) \\
& \simeq \sup _{z \in \mathbb{C}} \int_{D(z, r)}(1+|\zeta|)^{q}|f(\zeta)| d m(\zeta) \\
& \lesssim \sup _{z \in \mathbb{C}} \sup _{\zeta \in D(z, r)}(1+|\zeta|)^{q}|f(\zeta)| \leq\|f\|_{L_{\varphi_{q}}^{\infty}} .
\end{array}
$$

Seemingly, we also have

$$
\begin{aligned}
\sup _{z \in \mathbb{C}}\left|\tilde{f}_{q}(z)\right| \leq \sup _{z \in \mathbb{C}}(1+|z|)^{q} & \int_{\mathbb{C}}\left|k_{z}(\zeta)\right|^{q} e^{-\frac{q \alpha}{2}|\zeta|^{2}}|f(\zeta)| d m(\zeta) \\
& =\sup _{z \in \mathbb{C}}(1+|z|)^{q} \int_{\mathbb{C}} e^{-\frac{q \alpha}{2}|z-\zeta|^{2}}|f(\zeta)| d m(\zeta) \\
& =\sup _{z \in \mathbb{C}}(1+|z|)^{q} \int_{\mathbb{C}} e^{-\frac{q \alpha}{2}|\tau|^{2}}|f(z-\tau)| d m(\tau) .
\end{aligned}
$$

Now observe that since $f$ belongs to $L_{\varphi_{q}}^{\infty}$, it satisfies $|f(\zeta)| \lesssim(1+|\zeta|)^{-q}$ for each $\zeta$ in $\mathbb{C}$. This means that the last integral in (3.2) is uniformly bounded independent of $z$. If we set $M_{1}$ to be one of such a bound and

$$
M=\sup _{f \in L_{\varphi q}^{\infty}} \sup _{z \in \mathbb{C}}|f(z)|<\infty,
$$

then we see that the integral is indeed bounded by $M_{1} M^{2}|f(z)|$ for any $z$. Taking this into account, we obtain

$$
\begin{array}{r}
\sup _{z \in \mathbb{C}}\left|\widetilde{f}_{q}(z)\right| \leq \sup _{z \in \mathbb{C}}(1+|z|)^{q} \int_{\mathbb{C}} e^{-\frac{q \alpha}{2}|\tau|^{2}}|f(z-\tau)| d m(\tau) \\
\leq \sup _{z \in \mathbb{C}}(1+|z|)^{q} M_{1} M^{2}|f(z)| \simeq\|f\|_{L_{\varphi_{q}}^{\infty}}
\end{array}
$$

and completes the proof.

Lemma 3.3. Let $\mu$ be a nonnegative measure on $\mathbb{C}, \quad D_{r q \mu}(z)=(1+|z|)^{q} \mu(D(z, r))$ for positive values $r$ and $q$, and $0<p \leq \infty$. Then if $D_{\delta q \mu}$ belongs to $L^{p}$ for some $\delta>0$, then $D_{\text {rqu }}$ belongs to $L^{p}$ for all $r>0$. 
Proof. For each $\tau$ in $\mathbb{C}$ we may write

$$
\int_{D(\tau, r)}(1+|z|)^{q} \mu(D(z, \delta)) d m(z)=\int_{\mathbb{C}} \int_{\mathbb{C}}(1+|z|)^{q} \chi_{D(\tau, r)}(z) \chi_{D(z, \delta)}(\zeta) d \mu(\zeta) d m(z) .
$$

Using again the simple fact that $\chi_{D(z, \delta)}(\zeta)=\chi_{D(\zeta, \delta)}(z)$, the double integral above is easily seen to be equal to

$$
\begin{array}{r}
\int_{\mathbb{C}} \int_{D(\zeta, \delta) \cap D(\tau, r)}(1+|z|)^{q} d m(z) d \mu(\zeta) \\
\geq \int_{\mathbb{C}}(1+|\tau|)^{q} m(D(\zeta, \delta) \cap D(\tau, r)) d \mu(\zeta) \\
\geq(1+|\tau|)^{q} \int_{D(\tau, r)} m(D(\zeta, \delta) \cap D(\tau, r)) d \mu(\zeta),
\end{array}
$$

where $m(E)$ refers to the Lebesque area measure of set $E$. Clearly, the right hand quantity is bounded from below by

$$
(1+|\tau|)^{q} \mu(D(\tau, r)) \inf _{\zeta \in D(\tau, r)} m(D(\zeta, \delta) \cap D(\tau, r)) \gtrsim(1+|\tau|)^{q} \mu(D(\tau, r)),
$$

where the lower estimate follows since $\zeta \in D(\tau, r)$, there obviously exists a disc $D\left(\tau_{0}, r_{0}\right)$ contained in $D(\zeta, \delta) \cap D(\tau, r)$ with $m\left(D\left(\tau_{0}, r_{0}\right)\right)=\pi r_{0}^{2}$. From the above analysis, we conclude

$$
(1+|\tau|)^{q} \mu(D(\tau, r)) \lesssim \int_{D(\tau, r)}(1+|z|)^{q} \mu(D(z, \delta)) d m(z) .
$$

If we now set $f(z)=\mu(D(z, \delta))$, then the estimate above along with Lemma 3.2 ensure that

$$
\left\|D_{r q \mu}\right\|_{L^{p}} \lesssim\left\|f_{\delta}\right\|_{L^{p}} \lesssim\|f\|_{L_{\varphi q}^{p}}=\left\|D_{\delta q \mu}\right\|_{L^{p}}<\infty
$$

for each $p \geq 1$ and any $r>0$. We need to deduce the same when $0<p<1$. In this case, (3.3) and Fubini's theorem imply

$$
\begin{aligned}
\int_{\mathbb{C}}(1+|\tau|)^{p q}(\mu(D(\tau, r)))^{p} d m(\tau) & \lesssim \int_{\mathbb{C}}\left(\int_{D(\tau, r)}(1+|z|)^{q} \mu(D(z, \delta)) d m(z)\right)^{p} d m(\tau) \\
& \leq \int_{\mathbb{C}} \int_{D(\tau, r)}(1+|z|)^{p q}(\mu(D(z, \delta)))^{p} d m(z) d m(\tau) \\
& =\int_{\mathbb{C}} \int_{\mathbb{C}} \chi_{D(\tau, r)}(z) \frac{(\mu(D(z, \delta)))^{p}}{(1+|z|)^{-p q}} d m(z) d m(\tau) \\
& =\int_{\mathbb{C}}\left(\int_{\mathbb{C}} \chi_{D(z, r)}(\tau) d m(\tau)\right) \frac{(\mu(D(z, \delta)))^{p}}{(1+|z|)^{-p q}} d m(z) \\
& =\pi r^{2} \int_{\mathbb{C}}(1+|z|)^{p q}(\mu(D(z, \delta)))^{p} d m(z)<\infty
\end{aligned}
$$

independent of the choice of $r$ again. This along with (3.4) establishes our claim for all exponent $p$. 
We next recall the notion of lattice for the complex plane $\mathbb{C}$. For a positive $r$, we say that a sequence of distinct points $\left(z_{k}\right)_{k \in \mathbb{N}} \subset \mathbb{C}$ is an $r / 2$ - lattice for $\mathbb{C}$ if the sequence of the discs $D\left(z_{k}, r\right), k \in \mathbb{N}$ constitutes a covering of $\mathbb{C}$ and the discs $D\left(z_{k}, r / 2\right)$ are mutually disjoint. An interesting example of such a lattice can be found in [7].

Lemma 3.4. Let $r>0$ and $\left(z_{k}\right)_{k \in \mathbb{N}}$ be an $r / 2$ - lattice for $\mathbb{C}$. Then there exists a positive integer $N_{\max }$ such that every point in $\mathbb{C}$ belongs to at most $N_{\max }$ of the discs $D\left(z_{k}, 2 r\right)$.

The proof of the lemma can be found in [18, 24] where in [18] a more general setting has been considered. The sequence $z_{k}, k \in \mathbb{N}$ will refer to such a fixed $r / 2$ lattice in the remaining part of the paper. We now establish a basic lemma that will be used in the proof of the necessity parts of our main results. The lemma is also of its own interest.

Lemma 3.5. Let $\mu$ be a nonnegative measure on $\mathbb{C}, q \geq 0$ and $0<p \leq \infty$. Then the following statements are equivalent.

(i) The function $\widetilde{\mu}_{q}$ belongs to $L^{p}$;

(ii) $D_{\text {rqu }}$ belongs to $L^{p}$ for some (or any) $r>0$;

(iii) The sequence $\left(\left(\left|z_{j}\right|+1\right)^{q} \mu\left(D\left(z_{j}, r\right)\right)\right)_{j \in \mathbb{N}}$ belongs to $\ell^{p}$ for some (or any) $r>0$. Moreover, we have

$$
\left\|\widetilde{\mu}_{q}\right\|_{L^{p}} \simeq\left\|D_{r q \mu}\right\|_{L^{p}} \simeq\left\|\left(\left(1+\left|z_{j}\right|\right)^{q} \mu\left(D\left(z_{j}, r\right)\right)\right)\right\|_{l^{p}}
$$

Proof. We begin with the proof of (i) implies (ii). For any nonnegative $r$ and $q$, we have

$$
\begin{aligned}
D_{r q \mu}(z) & =(|z|+1)^{q} \int_{D(z, r)} d \mu(w) \\
& \lesssim \int_{D(z, r)}(|z|+1)^{q} e^{\frac{\alpha q}{2}\left(r^{2}-|w-z|^{2}\right)} d \mu(w) \lesssim \tilde{\mu}_{q}(z)
\end{aligned}
$$

from which we obtain the estimate

$$
\left\|D_{r q \mu}\right\|_{L^{p}} \lesssim\left\|\widetilde{\mu}_{q}\right\|_{L^{p}}<\infty
$$

independently of the choices of $r$ and exponent $p$.

We now prove the equivalency of statements (ii) and (iii), and assume that part (ii) holds. Note that by the triangle inequality

$$
\mu(D(z, 2 r)) \geq \mu\left(D\left(z_{j}, r\right)\right)
$$


for each $z$ in $D\left(z_{j}, r\right)$. This and Lemma 3.3 imply

$$
\begin{aligned}
& \infty>N_{\max } \int_{\mathbb{C}}\left(\frac{\mu(D(z, 2 r))}{(|z|+1)^{-q}}\right)^{p} d m(z) \geq \sum_{j=1}^{\infty} \int_{D\left(z_{j}, r\right)}\left(\frac{\mu(D(z, 2 r))}{(|z|+1)^{-q}}\right)^{p} d m(z) \\
& \geq \sum_{j=1}^{\infty} \int_{D\left(z_{j}, r\right)}\left((|z|+1)^{q} \mu\left(D\left(z_{j}, r\right)\right)\right)^{p} d m(z) \geq \frac{\pi r^{2}}{2} \sum_{j=1}^{\infty}\left(\frac{\mu(D(z, 2 r))}{(|z|+1)^{-q}}\right)^{p} .
\end{aligned}
$$

Since for each $z$, there exists $j$ for which $z$ belongs to the disc $D\left(z_{j}, r\right)$, the case $p=\infty$ follows easily from the relation in (3.7). Therefore,

$$
\left\|\left(\left(\left|z_{j}\right|+1\right)^{q} \mu\left(D\left(z_{j}, r\right)\right)\right)\right\|_{\ell^{p}} \lesssim\left\|D_{2 r q \mu}\right\|_{L^{p}} \lesssim\left\|D_{r q \mu}\right\|_{L^{p}}
$$

for all positive $r$ and all $p$.

Conversely, assume that the sequence $\left(\mu\left(D\left(z_{j}, r\right)\right)\left(1+\left|z_{j}\right|\right)^{q}\right)_{j \in \mathbb{N}}$ belongs to $\ell^{p}$. Then

$$
\begin{aligned}
\int_{\mathbb{C}} D_{r q \mu}^{p}(z) d m(z) & \simeq \sum_{j=1} \int_{D\left(z_{j}, r\right)}|\mu(D(z, r))|^{p}(1+|z|)^{q p} d m(z) \\
\leq & \sum_{j=1} \int_{D\left(z_{j}, r\right)}\left(\mu\left(D\left(z_{j}, r\right)\right)\right)^{p}(1+|z|)^{q p} d m(z) \\
& \simeq \sum_{j=1} \int_{D\left(z_{j}, r\right)}\left(\left(\mu\left(D\left(z_{j}, r\right)\right)\right)^{p}\left(1+\left|z_{j}\right|\right)^{q p} .\right.
\end{aligned}
$$

The case $p=\infty$ holds trivially again. Thus, from (3.9) we establish the estimate

$$
\left\|D_{r q \mu}\right\|_{L^{p}} \lesssim\left\|\left(\left(\left|z_{j}\right|+1\right)^{q} \mu\left(D\left(z_{j}, r\right)\right)\right)\right\|_{\ell^{p}} .
$$

To complete the proof of the lemma, we next show that (ii) implies (i). By Lemma 2.1 of [7] applied to the function $k_{w}$, we have

$$
\left|k_{w}(\zeta)\right|^{q} e^{-\frac{q \alpha}{2}|\zeta|^{2}} \lesssim \int_{D(\zeta, r)}\left|k_{w}(z)\right|^{q} e^{-\frac{q \alpha}{2}|z|^{2}} d m(z)
$$

Multiplying both sides of the inequality by $\varphi_{q}(w)$ and subsequently integrating against the measure $\mu$ give

$$
\begin{aligned}
\widetilde{\mu}_{q}(w) & =\int_{\mathbb{C}}(|w|+1)^{q}\left|k_{w}(\zeta)\right|^{q} e^{-\frac{q \alpha}{2}|\zeta|^{2}} d \mu(\zeta) \\
& \lesssim \int_{\mathbb{C}} \int_{D(\zeta, r)}(|w|+1)^{q}\left|k_{w}(z)\right|^{q} e^{-\frac{q \alpha}{2}|z|^{2}} d m(z) d \mu(\zeta) \\
& =\int_{\mathbb{C}}(|w|+1)^{q}\left|k_{w}(z)\right|^{q} e^{-\frac{q \alpha}{2}|z|^{2}} \int_{D(z, r)} d \mu(\zeta) d m(z) \\
& \leq \int_{\mathbb{C}}(|w|+1)^{q}\left|k_{w}(z)\right|^{q} e^{-\frac{q \alpha}{2}|z|^{2}} \mu(D(z, r)) d m(z)=\widetilde{f}_{q}(w),
\end{aligned}
$$


where we set $f(z)=\mu(D(z, r))$. This along with Lemma 3.2 yield

$$
\left\|\widetilde{\mu}_{q}\right\|_{L^{p}} \lesssim\left\|\widetilde{f}_{q}\right\|_{L^{p}} \lesssim\|f\|_{L_{\varphi q}^{p}}=\left\|D_{r q \mu}\right\|_{L^{p}}<\infty
$$

for all $p \geq 1$. To this end, we remain with the case for $p<1$. Observe that for each $z$ in $D\left(z_{j}, r\right)$, we estimate

$$
|z-w|^{2} \geq\left(\left|w-z_{j}\right|-\left|z-z_{j}\right|\right)^{2} \geq\left|w-z_{j}\right|^{2}-2 r\left|w-z_{j}\right| .
$$

From this fact and completing the square in the inner product from the kernel function, it follows that

$$
\begin{aligned}
\int_{\mathbb{C}}\left|\widetilde{\mu}_{q}(w)\right|^{p} d m(w) & =\int_{\mathbb{C}}\left(\int_{\mathbb{C}}(|w|+1)^{q}\left|k_{w}(z)\right|^{q} e^{-\frac{\alpha q}{2}|z|^{2}} d \mu(z)\right)^{p} d m(w) \\
& \lesssim \int_{\mathbb{C}}\left(\sum_{j=1}^{\infty} \int_{D\left(z_{j}, r\right)}(|w|+1)^{q}\left|k_{z}(w)\right|^{q} e^{-\frac{\alpha q}{2}|w|^{2}} d \mu(z)\right)^{p} d m(w) \\
& \leq \int_{\mathbb{C}}\left(\sum_{j=1}^{\infty} \int_{D\left(z_{j}, r\right)}(|w|+1)^{q} e^{-\frac{q \alpha}{2}\left|w-z_{j}\right|^{2}+r \alpha q\left|w-z_{j}\right|} d \mu(z)\right)^{p} d m(w) \\
& \leq \sum_{j=1}^{\infty}\left(\mu\left(D\left(z_{j}, r\right)\right)\right)^{p} \int_{\mathbb{C}}(|w|+1)^{p q} \frac{e^{r \alpha q p\left|w-z_{j}\right|}}{e^{-\frac{q p \alpha}{2}\left|w-z_{j}\right|^{2}} d m(w),}
\end{aligned}
$$

where the last inequality is possible since $0<p<1$. Applying the change of variables $\zeta=w-z_{j}$ and using the obvious inequality $|a+b|^{q} \leq 2^{q}\left(|a|^{q}+|b|^{q}\right)$, the integral above can be estimated as

$$
\begin{aligned}
\int_{\mathbb{C}}(|w|+1)^{p q} e^{-\frac{q p \alpha}{2}\left|w-z_{j}\right|^{2}+r \alpha q p\left|w-z_{j}\right|} d m(w) \\
=\int_{\mathbb{C}}\left(\left|\zeta+z_{j}\right|+1\right)^{p q} e^{-\frac{q p \alpha}{2}|\zeta|^{2}+r \alpha q p|\zeta|} d m(\zeta)
\end{aligned}
$$

which is bounded by

$$
2^{q p}\left(\left|z_{j}\right|+1\right)^{p q} \int_{\mathbb{C}}(|\zeta|+1)^{p q} e^{-\frac{q p \alpha}{2}|\zeta|^{2}+r \alpha q p|\zeta|} d m(\zeta) \lesssim\left(\left|z_{j}\right|+1\right)^{p q}
$$

as the last integral is easily seen to be convergent. Using this fact, relation (3.14), and the equivalency of the already proved statements in (ii) and (iii) in the lemma, we obtain

$$
\int_{\mathbb{C}}\left|\widetilde{\mu}_{q}(w)\right|^{p} d m(w) \lesssim \sum_{j=1}^{\infty}\left(\left|z_{j}\right|+1\right)^{p q}\left(\mu\left(D\left(z_{j}, r\right)\right)\right)^{p} \simeq\left\|D_{r q \mu}\right\|_{L^{p}}^{p}
$$

as required. Now from the series of estimates in (3.6), (3.8), (3.10), (3.13) and (3.15), we conclude the asymptotic norm relations in (3.5). 


\section{PRoOf OF THE MAIN RESUlts}

Before we begin proving the main results, we proceed to recall a few more facts that will be used in our subsequent considerations. A recent result of Constantin [5] ensures that for each entire function $f$

$$
\int_{\mathbb{C}}|f(z)|^{p} e^{-\frac{p \alpha}{2}|z|^{2}} d m(z) \simeq|f(0)|^{p}+\int_{\mathbb{C}}\left|f^{\prime}(z)\right|^{p}(1+|z|)^{-p} e^{-\frac{p \alpha}{2}|z|^{2}} d m(z)
$$

for $0<p<\infty$. The corresponding estimate for $p=\infty$ follows from [15] and reads

$$
\sup _{z \in \mathbb{C}}|f(z)| e^{-\frac{\alpha}{2}|z|^{2}} \simeq|f(0)|+\sup _{z \in \mathbb{C}}\left|f^{\prime}(z)\right|(1+|z|)^{-1} e^{-\frac{\alpha}{2}|z|^{2}} .
$$

These Littelwood-Paley type estimates provide a natural description of the Fock spaces $\mathcal{F}_{\alpha}^{p}$ in terms of the first derivatives and we will use them repeatedly in our proofs. From the relations in (4.1) and (4.2), we also easily deduce the pointwise estimate

$$
\left|f^{\prime}(z)\right| \lesssim(1+|z|) e^{\frac{\alpha}{2}|z|^{2}}\|f\|_{p}
$$

for each point $z$ in $\mathbb{C}$, exponent $0<p \leq \infty$, and holomorphic function $f$.

4.1. Proof of Theorem 2.1. (i) Sufficiency. First assume that $q<\infty$. Since $\left|f^{\prime}\right|^{p}$ is subharmonic for each holomorphic function $f$, by Lemma 1 of [8], we have the local estimate

$$
\left|f^{\prime}(z)\right|^{p} e^{-\frac{\alpha p}{2}|z|^{2}} \lesssim \int_{D(z, r)}\left|f^{\prime}(w)\right|^{p} e^{-\frac{\alpha p}{2}|w|^{2}} d m(w),
$$

where $r>0$ is fixed. Applying (4.1) and (4.4) with $p=q$ and $r=1$, we obtain

$$
\left\|J_{(g, \psi)} f\right\|_{q}^{q} \lesssim \int_{\mathbb{C}} e^{\frac{q \alpha}{2}\left(|\psi(z)|^{2}-|z|^{2}\right)} \frac{|g(z)|^{q}}{(1+|z|)^{q}} \int_{\mathbb{C}} \chi_{D(\psi(z), 1)}(w) \frac{\left|f^{\prime}(w)\right|^{q}}{e^{\frac{\alpha q}{2}|w|^{2}}} d m(w) d m(z) .
$$

On the other hand, since $\chi_{D(\psi(z), 1)}(w)=\chi_{D(w, 1)}(\psi(z))$, by Fubini's theorem it follows that the right-hand side of the above inequality is equal to

$$
\begin{aligned}
\int_{\mathbb{C}}\left|f^{\prime}(w)\right|^{q} e^{-\frac{\alpha q}{2}|w|^{2}} \int_{D(w, 1)} e^{\frac{q \alpha}{2}|\xi|^{2}} d \mu_{g}(\xi) d m(w) \\
\simeq \int_{\mathbb{C}}\left|f^{\prime}(w)\right|^{q} \frac{e^{-\frac{\alpha q}{2}|w|^{2}}}{(1+|w|)^{q}} \int_{D(w, 1)}(1+|\xi|)^{q} e^{\frac{q \alpha}{2}|\xi|^{2}} d \mu_{g}(\xi) d m(w)
\end{aligned}
$$

where we set $\xi=\psi(z)$,

$$
d \mu_{g}(E)=\int_{\psi^{-1}(E)} \frac{|g(z)|^{q}}{(1+|z|)^{q}} e^{-\frac{\alpha q}{2}|z|^{2}} d m(z)
$$


for every Borel subset $E$ of $\mathbb{C}$, and use the fact that $1+|w| \simeq 1+|\xi|$ whenever $\xi$ belongs to the disc $D(w, 1)$. Applying (4.1) with $p=q$ again, the right-hand double integral in (4.6) is bounded by a constant multiple of

$$
\|f\|_{q}^{q} \sup _{w \in \mathbb{C}} \int_{D(w, 1)}(1+|\xi|)^{q} e^{\frac{q \alpha}{2}|\xi|^{2}} d \mu_{g}(\xi) .
$$

If we show that the supremum above is finite, then the desired conclusion follows since $\mathcal{F}_{\alpha}^{p} \subseteq \mathcal{F}_{\alpha}^{q}$ whenever $p \leq q$. To this end, we have

$$
\begin{aligned}
\sup _{w \in \mathbb{C}} \int_{D(w, 1)}(1+|\xi|)^{q} e^{\frac{q \alpha}{2}|\xi|^{2}} d \mu_{g}(\xi) & \simeq \sup _{w \in \mathbb{C}}(1+|w|)^{q} \int_{D(w, 1)} e^{\frac{q \alpha}{2}|\xi|^{2}} d \mu_{g}(\xi) \\
& \lesssim \sup _{w \in \mathbb{C}} B_{\left(|g|^{q}, \psi\right)}(w),
\end{aligned}
$$

where in the last relationship we have used a simple fact that if $\xi \in D(w, 1)$, then

$$
\left|k_{w}(\xi)\right|^{q}=\left|e^{-\frac{\alpha}{2}|w|^{2}+\alpha \bar{w} \xi}\right|^{q}=e^{\frac{q \alpha}{2}\left(|\xi|^{2}-|\xi-w|^{2}\right)} \gtrsim e^{\frac{q \alpha}{2}|\xi|^{2}},
$$

and integrating (4.8) against the measure $\mu_{g}$ we have that

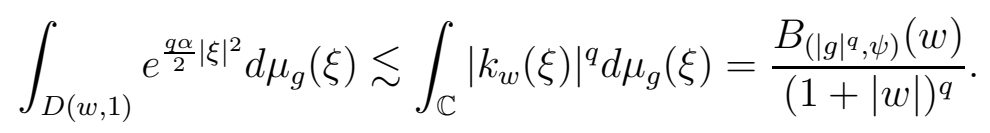

On the other hand, for $q=\infty$, the sufficiency of the condition follows from a simple estimation along with (4.2) and (4.3) as

$$
\begin{aligned}
\left\|J_{(g, \psi)} f\right\|_{\infty} & \simeq \sup _{z \in \mathbb{C}} \frac{\left|f^{\prime}(\psi(z))\right||g(z)|}{(1+|z|)} e^{-\frac{\alpha}{2}|z|^{2}} \\
& \lesssim\|f\|_{p} \sup _{z \in \mathbb{C}} \frac{(|\psi(z)|+1)|g(z)|}{1+|z|} e^{\frac{\alpha}{2}\left(|\psi(z)|^{2}-|z|^{2}\right)}=\|f\|_{p}\left\|M_{(g, \psi)}\right\|_{L^{\infty}} .
\end{aligned}
$$

From the series of estimations (4.5), (4.6), (4.7) and (4.9), we have already one side of the asymptotic relation in (2.1), namely that $\left\|J_{(g, \psi)}\right\|$ is bounded by a constant multiple of $\left\|M_{(g, \psi)}\right\|_{L^{\infty}}$ for $q=\infty$ and $\left\|B_{\left(|g|^{q}, \psi\right)}\right\|_{L^{\infty}}^{1 / q}$ whenever $q<\infty$.

Necessity. Assuming that $q<\infty$, we apply $J_{(g, \psi)}$ to $k_{w}$ and invoke (4.1) to obtain

$$
1 \gtrsim\left\|J_{(g, \psi)} k_{w}\right\|_{q}^{q} \simeq \int_{\mathbb{C}}\left|w k_{w}(\psi(z))\right|^{q} \frac{|g(z)|^{q}}{(1+|z|)^{q}} e^{-\frac{\alpha q}{2}|z|^{2}} d m(z)=S_{1} .
$$

To arrive at the desired conclusion, it remains to show the estimate

$$
\left\|J_{(g, \psi)} k_{w}\right\|_{q}^{q} \gtrsim \int_{\mathbb{C}}\left|k_{w}(\psi(z))\right|^{q} \frac{|g(z)|^{q}}{(1+|z|)^{q}} e^{-\frac{\alpha q}{2}|z|^{2}} d m(z)=S_{2} .
$$

To do so, we may invoke Lemma 3.5 and equivalently express the conditions in the theorem in terms of the sequence $\left(\left(1+\left|z_{j}\right|\right)^{q} \mu\left(D\left(z_{j}, r\right)\right)\right)_{j \in \mathbb{N}}$ for some measure $\mu$ 
on $\mathbb{C}$. Indeed, carefully examining the proof of the lemma we see that the sequence $\left(\left|z_{j}\right|^{q} \mu\left(D\left(z_{j}, r\right)\right)\right)_{j \in \mathbb{N}}$ belongs to $\ell^{p}$ if and only if the function

$$
\widehat{\mu}_{q}(w)=\int_{\mathbb{C}}|w|^{q}\left|k_{w}(z)\right|^{q} e^{-\frac{\alpha q}{2}|z|^{2}} d \mu(z)
$$

belongs to $L^{p}$. Using this fact for the case $p=\infty$, we may equivalently write condition (4.10) as

$$
\sup _{j \in \mathbb{N}}\left|z_{j}\right|{ }^{q} \mu_{(g, \psi)}\left(D\left(z_{j}, r\right)\right) \simeq\left\|J_{(g, \psi)} k_{w}\right\|_{q}^{q} \lesssim 1,
$$

where $\mu_{(g, \psi)}$ is a measure on $\mathbb{C}$ such that

$$
d \mu_{(g, \psi)}(z)=\frac{|g(z)|^{q}}{(1+|z|)^{q}} e^{\frac{\alpha q}{2}\left(|\psi(z)|^{2}-|z|^{2}\right)} d m(z) \circ \psi^{-1}(z) .
$$

Having singled out this connection with a discrete setting, we will arrive at (4.11) if we equivalently show that the sequence

$$
\sup _{j \in \mathbb{N}} \mu_{(g, \psi)}\left(D\left(z_{j}, r\right)\right)<\infty .
$$

This immediately follows from (4.12) if $\left|z_{j}\right| \geq 1$ for all $j$ in $\mathbb{N}$. On the other hand, since the sequence $\left(z_{j}\right), j \in \mathbb{N}$ is an $r / 2$ - lattice for $\mathbb{C},\left|z_{j}\right| \rightarrow \infty$ as $j \rightarrow \infty$. This means that the inequality $\left|z_{j}\right|<1$ can possibly hold for only a finite number of indices $j$, and hence

$$
\sup _{j \in \mathbb{N},\left|z_{j}\right|<1} \mu_{(g, \psi)}\left(D\left(z_{j}, r\right)\right) \lesssim \sup _{j \in \mathbb{N},\left|z_{j}\right| \geq 1} \mu_{(g, \psi)}\left(D\left(z_{j}, r\right)\right) \lesssim\left\|J_{(g, \psi)} k_{w}\right\|_{q}^{q}
$$

from which and (4.10) we conclude that

$$
\left\|J_{(g, \psi)} k_{w}\right\|_{q}^{q} \gtrsim S_{1}+S_{2} \gtrsim B_{\left(|g|^{q}, \psi\right)}(w) .
$$

On the other hand, if $q=\infty$, then applying (4.2) we have

$$
\begin{aligned}
\left\|J_{(g, \psi)}\right\| \gtrsim\left\|J_{(g, \psi)} k_{w}\right\|_{\infty} & \simeq \sup _{z \in \mathbb{C}} \frac{|g(z)|}{1+|z|}\left|\bar{w} k_{w}(\psi(z))\right| e^{-\frac{\alpha}{2}|z|^{2}} \\
& \geq \frac{|g(z) \bar{w}|}{1+|z|}\left|e^{\alpha \bar{w} \psi(z)}\right| e^{-\frac{\alpha}{2}\left(|w|^{2}+|z|^{2}\right)}
\end{aligned}
$$

for all $z$ and $w$ in $\mathbb{C}$. In particular, when we set $w=\psi(z)$, we get

$$
\left\|J_{(g, \psi)}\right\| \gtrsim\left\|J_{(g, \psi)} k_{w}\right\|_{\infty} \gtrsim \frac{|\psi(z)||g(z)|}{1+|z|} e^{\frac{\alpha}{2}\left(|\psi(z)|^{2}-|z|^{2}\right)} .
$$

From (4.14) we have that

$$
e^{\frac{\alpha}{2}\left(|\psi(z)|^{2}-|z|^{2}\right)} \lesssim \frac{|z|+1}{M_{\infty}(g \psi,|z|)}
$$


whenever $g \psi$ is a nonzero function. Estimate (4.15) along with the fact that the integral mean $M_{\infty}(g \psi,|z|)$ is a nondecreasing function of $|z|$, implies that

$$
\limsup _{|z| \rightarrow \infty}(|\psi(z)|-|z|) \leq 0
$$

Otherwise, there would be a sequence $\left(w_{j}\right)_{j \in \mathbb{N}}$ such that $\left|w_{j}\right| \uparrow \infty$ as $j \rightarrow \infty$, and

$$
\limsup _{j \rightarrow \infty}\left(\left|\psi\left(w_{j}\right)\right|-\left|w_{j}\right|\right)=a>0,
$$

from which and (4.15) we would have that the sequence $e^{\frac{\alpha}{2}\left(\left|\psi\left(w_{j}\right)\right|^{2}-\left|w_{j}\right|^{2}\right)} /\left(\left|w_{j}\right|+1\right)$, $j \in \mathbb{N}$, is unbounded, and contradicts (4.15).

From (4.16) and the Cauchy inequality we easily obtain that $\psi$ has the form $\psi(z)=$ $a z+b$ with $|a| \leq 1$ and $b=0$ whenever $|a|=1$. Using this in (4.14) we have that

$$
\left\|J_{(g, \psi)}\right\| \gtrsim \sup _{z \in \mathbb{C}} \frac{|a z+b||g(z)|}{1+|z|} e^{\frac{\alpha}{2}\left(|\psi(z)|^{2}-|z|^{2}\right)},
$$

from which it easily follows that

$$
\left\|J_{(g, \psi)}\right\| \gtrsim \sup _{z \in \mathbb{C}} \frac{(|\psi(z)|+1)|g(z)|}{1+|z|} e^{\frac{\alpha}{2}\left(|\psi(z)|^{2}-|z|^{2}\right)}=M_{(g, \psi)}(z) .
$$

(ii) We now assume that $B_{\left(|g|^{q}, \psi\right)}$ vanishes at infinity and proceed to prove that $J_{(g, \psi)}$ is compact. We consider a sequence $\left(f_{n}\right)_{n \in \mathbb{N}}$ of functions in $\mathcal{F}_{\alpha}^{p}$ such that $\sup _{n}\left\|f_{n}\right\|_{p}<\infty$ and $f_{n}$ converges to zero uniformly on compact subsets of $\mathbb{C}$ as $n \rightarrow \infty$. Following the arguments made in the proof of the sufficiency part, for a positive $R$ and a finite exponent $q \geq p$, we have

$$
\begin{aligned}
\limsup _{n \rightarrow \infty}\left\|J_{(g, \psi)} f_{n}\right\|_{q}^{q} & \lesssim \limsup _{n \rightarrow \infty} \int_{\mathbb{C}} \frac{\left|f_{n}^{\prime}(w)\right|^{q}}{(1+|w|)^{q}} e^{-\frac{\alpha q}{2}|w|^{2}} B_{\left(|g|^{q}, \psi\right)}(w) d m(w) \\
\leq & \limsup _{n \rightarrow \infty} \int_{|w| \leq R} \frac{\left|f_{n}^{\prime}(w)\right|^{q} e^{-\frac{\alpha q}{2}|w|^{2}}}{(1+|w|)^{q}} B_{\left(|g|^{q}, \psi\right)}(w) d m(w) \\
& +\limsup _{n \rightarrow \infty} \sup _{|w|>R} B_{\left(|g|^{q}, \psi\right)}(w) \int_{|w|>R} \frac{\left|f_{n}^{\prime}(w)\right|^{q} e^{-\frac{\alpha q}{2}|w|^{2}}}{(1+|w|)^{q}} d m(w),
\end{aligned}
$$

for every $R>0$.

Since $B_{\left(|g|^{q}, \psi\right)}$ vanishes at infinity we have that for every $\varepsilon>0$ there is an $R_{0}>0$ such that

$$
\sup _{|w|>\rho} B_{\left(|g|^{q}, \psi\right)}(w)<\varepsilon
$$

for every $\rho \geq R_{0}$. We may assume that $R_{0}=R$. 
Applying (4.1) and the assumption that $\left\|f_{n}\right\|_{p}$ is uniformly bounded, we obtain

$$
\limsup _{n \rightarrow \infty} \sup _{|w|>R} B_{\left(|g|^{q}, \psi\right)}(w) \int_{|w|>R} \frac{\left|f_{n}^{\prime}(w)\right|^{q} e^{-\frac{\alpha q}{2}|w|^{2}}}{(1+|w|)^{q}} d m(w) \lesssim \sup _{|w|>R} B_{\left(|g|^{q}, \psi\right)}(w)<\varepsilon .
$$

On the other hand, we have the next estimate

$$
\int_{|z| \leq R} \frac{\left|f_{n}^{\prime}(w)\right|^{q} B_{\left(|g|^{q}, \psi\right)}(w)}{(1+|w|)^{q} e^{\frac{\alpha q}{2}|w|^{2}}} d m(w) \lesssim \sup _{|w| \leq R}\left|f_{n}^{\prime}(w)\right|^{q} \lesssim \sup _{|w| \leq 2 R}\left|f_{n}(w)\right|^{q},
$$

where we have used the fact that the integral

$$
\int_{|w| \leq R} e^{-\frac{\alpha q}{2}|w|^{2}} B_{\left(|g|^{q}, \psi\right)}(w) d m(w)
$$

is finite due to the boundedness of $B_{\left(|g|^{q}, \psi\right)}(w)$. Since $f_{n}$ converges to zero uniformly on compact subsets of $\mathbb{C}$, taking the lim sup in (4.21) and combining the result with (4.20) we get

$$
\limsup _{n \rightarrow \infty}\left\|J_{(g, \psi)} f_{n}\right\|_{q}^{q} \lesssim \varepsilon .
$$

From this and since $\varepsilon$ is an arbitrary positive number we get $\lim _{n \rightarrow \infty}\left\|J_{(g, \psi)} f_{n}\right\|_{q}=0$, so by Lemma 3.1 the compactness follows.

We need to conclude the same when $q=\infty$. For this, we may modify a common approach used in dealing with the compactness of operators acting between spaces of holomorphic function with target space restricted to be a growth space (see, for example, $[15,20,21])$. To this end, we note that the function $f_{0}(z)=z$ belongs to $\mathcal{F}_{\alpha}^{p}$ for all $p>0$. It follows that by the boundedness and (4.2),

$$
\left\|J_{(g, \psi)} f_{0}\right\|_{\infty} \simeq \sup _{z \in \mathbb{C}}|g(z)|(1+|z|)^{-1} e^{-\frac{\alpha}{2}|z|^{2}}<\infty .
$$

For each positive $\epsilon$, the necessity of the condition implies that there exists a positive $N_{1}$ such that

$$
M_{(g, \psi)}(z)<\epsilon
$$

for all $z \in \mathbb{C}$ such that $|\psi(z)|>N_{1}$. From this along with (4.2) and (4.3), we get

$$
\frac{|g(z)|\left|f_{n}^{\prime}(\psi(z))\right|}{e^{\frac{\alpha}{2}|z|^{2}}(1+|z|)} \lesssim\left\|f_{n}\right\|_{p} \frac{\mid g(z)(|\psi(z)|+1)}{1+|z|} e^{\frac{\alpha}{2}|\psi(z)|^{2}-\frac{\alpha}{2}|z|^{2}} \lesssim M_{(g, \psi)}(z)<\epsilon
$$

for all $z \in \mathbb{C}$ such that $|\psi(z)|>N_{1}$ and all $n$. On the other hand if $|\psi(z)| \leq N_{1}$, then applying (4.22) it is easily seen that

$$
\frac{|g(z)|}{1+|z|}\left|f_{n}(\psi(z))\right| e^{-\frac{\alpha}{2}|z|^{2}} \lesssim \sup _{\left\{z:|\psi(z)| \leq N_{1}\right\}}\left|f_{n}(\psi(z))\right| \rightarrow 0
$$

as $n \rightarrow \infty$. Then we combine (4.23) and (4.24) to arrive at the desired conclusion.

To complete the proof of part (ii) of Theorem 2.1 , it remains to verify the necessity of 
the compactness condition. Since $k_{z}$ is bounded in $\mathcal{F}_{\alpha}^{p}$ and uniformly converges to zero on any compact subsets of $\mathbb{C}$ as $|z| \rightarrow \infty$, the compactness of $J_{(g, \psi)}$ implies

$$
B_{\left(|g|^{q}, \psi\right)}(z) \lesssim\left\|J_{(g, \psi)} k_{z}\right\|_{q}^{q} \rightarrow 0
$$

as $|z| \rightarrow \infty$ whenever $q$ is finite.

Now suppose that $q=\infty$ and further assume that there exists a sequence of points $\left(z_{j}\right)_{j \in \mathbb{N}} \subset \mathbb{C}$ such that $\left|\psi\left(z_{j}\right)\right| \rightarrow \infty$ as $j \rightarrow \infty$. If such a sequence does not exist, then necessity trivially holds. It follows from the compactness of $J_{(g, \psi)}$ that

$$
\limsup _{j \rightarrow \infty} M_{(g, \psi)}\left(z_{j}\right) \lesssim \limsup _{j \rightarrow \infty}\left\|J_{(g, \psi)} k_{z_{j}}\right\|_{\infty}=0,
$$

and completes the proof of part (ii) of Theorem 2.1.

The statements in parts (iii) and (iv) of the theorem follow from simple variants of the proofs of the statements in parts (i) and (ii) respectively. First observe that $\left(C_{(g, \psi)} f\right)^{\prime}(z)=$ $f^{\prime}(\psi(z)) g(\psi(z)) \psi^{\prime}(z)$. It means that we only need to replace the quantity $g(z)$ by $g(\psi(z)) \psi^{\prime}(z)$ in all the above arguments and proceed as in the preceding parts. Thus we omit the remaining details.

4.2. Proof of Theorem 2.2. (i). Since the compactness obviously implies the boundedness through the normal family argument, we will prove that boundedness implies the $L^{p /(p-q)}$ and $L^{1}$ integrability conditions and this in turn implies compactness. Proceeding as in the proof of the first part of Theorem 2.1, we have

$$
\begin{array}{r}
\left\|J_{(g, \psi)} f\right\|_{q}^{q} \lesssim \int_{\mathbb{C}} \frac{\left.\left|f^{\prime}(w)\right|\right|^{q} e^{-\frac{q \alpha}{2}|w|^{2}}}{(1+|w|)^{q}} \int_{D(w, 1)}(1+|\xi|)^{q} e^{\frac{q \alpha}{2}|\xi|^{2}} d \mu_{g}(\xi) d m(w) \\
\lesssim \int_{\mathbb{C}} \frac{\left|f^{\prime}(w)\right|^{q} e^{-\frac{q \alpha}{2}|w|^{2}}}{(1+|w|)^{q}} B_{\left(|g|^{q}, \psi\right)}(w) d m(w) .
\end{array}
$$

Since $p>q$, applying Hölder's inequality, the right hand side quantity is bounded by

$$
\begin{aligned}
\left(\int_{\mathbb{C}} \frac{\left|f^{\prime}(w)\right|^{p} e^{-\frac{p \alpha}{2}|w|^{2}}}{(1+|w|)^{p}} d m(w)\right)^{\frac{q}{p}} & \left.\left(\int_{\mathbb{C}}\left|B_{\left(|g|^{q}, \psi\right)}(w)\right|^{\frac{p}{p-q}} d m(w)\right)\right)^{\frac{p-q}{p}} d m(w) \\
& \left.\lesssim\|f\|_{p}^{q}\left(\int_{\mathbb{C}}\left|B_{\left(|g|^{q}, \psi\right)}(w)\right|^{\frac{p}{p-q}} d m(w)\right)\right)^{\frac{p-q}{p}}
\end{aligned}
$$

whenever $p$ is finite. On the other hand if $p=\infty$, then by (4.2), it follows that

$$
\begin{aligned}
\left\|J_{(g, \psi)} f\right\|_{q}^{q} & \lesssim \int_{\mathbb{C}} \frac{\left|f^{\prime}(w)\right|^{q} e^{-\frac{q \alpha}{2}|w|^{2}}}{(1+|w|)^{q}} B_{\left(|g|^{q}, \psi\right)}(w) d m(w) \\
& \lesssim\|f\|_{\infty}^{q} \int_{\mathbb{C}} B_{\left(|g|^{q}, \psi\right)}(w) d m(w) .
\end{aligned}
$$


From the estimates in (4.26), (4.27) and (4.28), we also have

$$
\left\|J_{(g, \psi)}\right\|^{q} \lesssim \begin{cases}\left\|B_{\left(|g|^{q}, \psi\right)}\right\|_{L^{\frac{p}{p-q}}}, & p<\infty \\ \left\|B_{\left(|g|^{q}, \psi\right)}\right\|_{L^{1}}, & p=\infty\end{cases}
$$

This establishes one part of the asymptotic relation in (2.3). The remaining part of the estimate will follow from our next proof of the integrability condition. For this, we appeal to the atomic decomposition of functions in Fock spaces, i.e., each function in $\mathcal{F}_{\alpha}^{p}$ is generated by an $\ell^{p}$ sequence as

$$
f=\sum_{j=1}^{\infty} c_{j} k_{z_{j}}, \text { and }\|f\|_{p} \simeq\left\|\left(c_{j}\right)\right\|_{\ell^{p}} .
$$

This was proved in [9] for $p \geq 1$ and in [23] for $0<p<1$. We first assume that $0<q<\infty$, and if $\left(r_{j}(t)\right)_{j \in \mathbb{N}}$ is the Rademacher sequence of functions on $[0,1]$ chosen as in [13], then Khinchine's inequality yields

$$
\left(\sum_{j=1}^{\infty}\left|c_{j} z_{j}\right|^{2}\left|k_{z_{j}}(z)\right|^{2}\right)^{\frac{q}{2}} \lesssim \int_{0}^{1}\left|\sum_{j=1}^{\infty} c_{j} z_{j} r_{j}(t) k_{z_{j}}(z)\right|^{q} d t .
$$

Note that here if the $r_{j}(t)$ 's are chosen as refereed above, then the sequence $\left(c_{j} r_{j}(t)\right)_{j \in \mathbb{N}}$ belongs to $\ell^{p}$ with $\left\|\left(c_{j} r_{j}(t)\right)\right\|_{\ell^{p}}=\left\|\left(c_{j}\right)\right\|_{\ell^{p}}$ for all $t$ and

$$
\sum_{j=1}^{\infty} c_{j} r_{j}(t) k_{z_{j}}(z) \in \mathcal{F}_{\alpha}^{p} \text {, with }\left\|\sum_{j=1}^{\infty} c_{j} r_{j}(t) k_{z_{j}}(z)\right\|_{p} \simeq\left\|\left(c_{j}\right)\right\|_{\ell^{p}} .
$$

Setting as before

$$
d \mu_{(g, \psi)}(z)=\frac{|g(z)|^{q}}{(1+|z|)^{q}} e^{\frac{\alpha q}{2}\left(|\psi(z)|^{2}-|z|^{2}\right)} d m(z) \circ \psi^{-1}(z)
$$

and making use of (4.31), and subsequently Fubini's theorem, we obtain

$$
\begin{aligned}
\int_{\mathbb{C}}\left(\sum_{j=1}^{\infty}\left|c_{j} z_{j}\right|^{2}\left|k_{z_{j}}(z)\right|^{2}\right)^{\frac{q}{2}} d \mu_{(g, \psi)}(z) & \lesssim \int_{\mathbb{C}}\left(\int_{0}^{1}\left|\sum_{j=1}^{\infty} c_{j} z_{j} r_{j}(t) k_{z_{j}}(z)\right|^{q} d t\right) d \mu_{(g, \psi)}(z) \\
& =\int_{0}^{1}\left(\int_{\mathbb{C}}\left|\sum_{j=1}^{\infty} c_{j} z_{j} r_{j}(t) k_{z_{j}}(z)\right|^{q} d \mu_{(g, \psi)}(z)\right) d t .
\end{aligned}
$$

Invoking (4.1) with $p=q$, the double integral above is asymptotically equal to

$$
\int_{0}^{1}\left\|J_{(g, \psi)} \sum_{j=1}^{\infty} c_{j} r_{j}(t) k_{z_{j}}\right\|_{q}^{q} d t \lesssim\left\|J_{(g, \psi)}\right\|^{q}\left\|\left(c_{j}\right)\right\|_{\ell^{p}}^{q},
$$

where the estimate follows by the boundedness assumption and (4.32). 
Now if $q \geq 2$, then we obviously have

$$
\sum_{j=1}^{\infty}\left|c_{j}\right|^{q}\left|z_{j}\right|^{q} \mu_{(g, \psi)}\left(D\left(z_{j}, r\right)\right) \lesssim \int_{\mathbb{C}}\left(\sum_{j=1}^{\infty}\left|c_{j}\right|^{2}\left|z_{j}\right|^{2} \chi_{D\left(z_{j}, r\right)}(z)\right)^{\frac{q}{2}} d \mu_{(g, \psi)}(z) .
$$

On the other hand, if $q<2$, then applying Hölder's inequality, we obtain

$$
\begin{aligned}
\sum_{j=1}^{\infty}\left|c_{j}\right|^{q}\left|z_{j}\right|^{q} \mu_{(g, \psi)}\left(D\left(z_{j}, r\right)\right) \leq( & \left.N_{\max }\right)^{\frac{2-q}{2}} \int_{\mathbb{C}}\left(\sum_{j=1}^{\infty}\left|c_{j}\right|^{2}\left|z_{j}\right|^{2} \chi_{D\left(z_{j}, r\right)}(z)\right)^{\frac{q}{2}} d \mu_{(g, \psi)}(z) \\
& \lesssim \int_{\mathbb{C}}\left(\sum_{j=1}^{\infty}\left|c_{j}\right|^{2}\left|z_{j}\right|^{2} \chi_{D\left(z_{j}, r\right)}(z)\right)^{\frac{q}{2}} d \mu_{(g, \psi)}(z) .
\end{aligned}
$$

From (4.34), (4.35), (4.36), and (4.37) we deduce

$$
\sum_{j=1}^{\infty}\left|c_{j}\right|^{q}\left|z_{j}\right|^{q} \mu_{(g, \psi)}\left(D\left(z_{j}, r\right)\right) \lesssim\left\|J_{(g, \psi)}\right\|^{q}\left\|\left(c_{j}\right)\right\|_{\ell^{p}}^{q}
$$

for each $q$.

Now, if $p=\infty$, we set $c_{j}=1$ for all $j \in \mathbb{N}$ in (4.38) to see that the sequence $\left|z_{j}\right|^{q} \mu\left(D\left(z_{j}, r\right)\right) \in \ell^{1}$. But we need to show that

$$
\sum_{j=1}^{\infty}\left(\left|z_{j}\right|+1\right)^{q} \mu_{(g, \psi)}\left(D\left(z_{j}, r\right)\right) \lesssim\left\|J_{(g, \psi)}\right\|^{q} .
$$

This obviously holds when $\left|z_{j}\right| \geq 1$ for all $j \in \mathbb{N}$. Thus, we proceed to verify the case when $\left|z_{j}\right|<1$. To this end, note that since $\left(z_{j}\right)_{j \in \mathbb{N}}$ is a fixed sequence with the property that $\left|z_{j}\right| \rightarrow \infty$ as $j \rightarrow \infty$, the inequality $\left|z_{j}\right|<1$ can happen only for a finite number of $j$ 's. It follows that there exist a positive constant $N_{f}$ for which

$$
\sum_{j:\left|z_{j}\right|<1} \frac{\mu_{(g, \psi)}\left(D\left(z_{j}, r\right)\right)}{\left(\left|z_{j}\right|+1\right)^{-q}} \leq N_{f} \sum_{j:\left|z_{j}\right| \geq 1} \frac{\mu_{(g, \psi)}\left(D\left(z_{j}, r\right)\right)}{\left(\left|z_{j}\right|+1\right)^{-q}} \lesssim\left\|J_{(g, \psi)}\right\|^{q} .
$$

On the other hand, if $p<\infty$, then since $\left(\left|c_{j}\right|^{q}\right)_{j \in \mathbb{N}} \in \ell^{p / q}$ a duality argument with (4.38) ensures that the sequence $\left(\left|z_{j}\right|^{q} \mu\left(D\left(z_{j}, r\right)\right)\right)_{j \in \mathbb{N}}$ belongs to $\ell^{p /(p-q)}$. Using this fact and following the same arguments made above for the case when $p=\infty$, we deduce

$$
\left(\left(\left|z_{j}\right|+1\right)^{q} \mu_{(g, \psi)}\left(D\left(z_{j}, r\right)\right)\right)_{j \in \mathbb{N}} \in \ell^{p /(p-q)} .
$$

We combine this with Lemma 3.5 to arrive at the desired conclusion. Looking at the above proof, we also have

$$
\left\|J_{(g, \psi)}\right\|^{q} \gtrsim\left\{\begin{array}{l}
\left\|\left(\left(\left|z_{j}\right|+1\right)^{q} \mu_{(g, \psi)}\left(D\left(z_{j}, r\right)\right)\right)\right\|_{\ell^{p /(p-q)}}, \quad p<\infty \\
\left\|\left(\left(\left|z_{j}\right|+1\right)^{q} \mu_{(g, \psi)}\left(D\left(z_{j}, r\right)\right)\right)\right\|_{\ell^{1}}, \quad p=\infty
\end{array}\right.
$$


from which and (3.5), the reverse asymptotic estimate in (4.29) holds.

It remains to prove that $L^{p /(p-q)}$ for $p<\infty$ and $L^{1}$ for $p=\infty$ integrability of $\widetilde{\mu}_{q}$ implies compactness of $J_{(g, \psi)}: \mathcal{F}_{\alpha}^{p} \rightarrow \mathcal{F}_{\alpha}^{q}$ whenever $q<p$. To this end, let $f_{n}$ be a sequence of functions in $\mathcal{F}_{\alpha}^{p}$ such that $\sup _{n}\left\|f_{n}\right\|_{p}<\infty$ and $f_{n}$ converges to zero uniformly on compact subset of $\mathbb{C}$ as $n \rightarrow \infty$. Assume $p<\infty$. Then for a positive $R$, replacing $f$ by $f_{n}$ in (4.26) we write

$$
\begin{gathered}
\left\|J_{(g, \psi)} f_{n}\right\|_{q}^{q} \lesssim\left(\int_{|w| \leq R}+\int_{|w|>R}\right) \frac{\left.\left|f_{n}^{\prime}(w)\right|\right|^{q} e^{-\frac{q \alpha}{2}|w|^{2}}}{(1+|w|)^{q}} B_{\left(|g|^{q}, \psi\right)}(w) d m(w) \\
=I_{n 1}+I_{n 2} .
\end{gathered}
$$

Applying Hölder's inequality, we estimate the second piece of integral as

$$
\begin{aligned}
I_{n 2}=\int_{|w|>R} \frac{\left|f_{n}^{\prime}(w)\right|^{q} e^{-\frac{q \alpha}{2}|w|^{2}}}{(1+|w|)^{q}} & B_{\left(|g|^{q}, \psi\right)}(w) d m(w) \\
& \leq\left\|f_{n}\right\|_{p}^{q}\left(\int_{|w|>R} B_{\left(|g|^{q}, \psi\right)}^{\frac{p}{p-q}}(w) d m(w)\right)^{\frac{p-q}{p}} \\
& \lesssim\left(\int_{|w|>R} B_{\left(|g|^{q}, \psi\right)}^{\frac{p}{p-q}}(w) d m(w)\right)^{\frac{p-q}{p}} \rightarrow 0
\end{aligned}
$$

when $R \rightarrow \infty$ since $B_{\left(|g|^{q}, \psi\right)}$ is $L^{p /(p-q)}$ integrable. On the other hand, because of this integrability and Hölder's inequality again, we have

$$
I_{n 1}=\int_{|w| \leq R} \frac{\left|f_{n}^{\prime}(w)\right|^{q} e^{-\frac{q \alpha}{2}|w|^{2}}}{(1+|w|)^{q}} B_{\left(|g|^{q}, \psi\right)}(w) d m(w) \lesssim\left(\int_{|w| \leq R} \frac{\left|f_{n}^{\prime}(w)\right|^{p} e^{-\frac{q \alpha}{2}|w|^{2}}}{(1+|w|)^{p}} d m(w)\right)^{\frac{q}{p}}
$$

Now for sufficiently large $R$, by (4.1), we have that

$$
\begin{array}{r}
I_{n 1} \lesssim\left(\int_{|w| \leq R} \frac{\left|f_{n}^{\prime}(w)\right|^{p} e^{-\frac{q \alpha}{2}|w|^{2}}}{(1+|w|)^{p}} d m(w)\right)^{\frac{q}{p}} \simeq\left(\int_{|w| \leq R}\left|f_{n}(w)\right|^{p} e^{-\frac{p \alpha}{2}|w|^{2}} d m(w)\right)^{\frac{q}{p}} \\
\lesssim \sup _{|w| \leq R}\left|f_{n}(w)\right|^{q} \rightarrow 0
\end{array}
$$

as $n \rightarrow \infty$ since $f_{n}$ converges to zero uniformly on compact subsets of $\mathbb{C}$.

Similarity, when $p=\infty$, by (4.3) and since $\left\|f_{n}\right\|_{\infty}$ is uniformly bounded, it follows that

$$
I_{n 2} \leq\left\|f_{n}\right\|_{\infty}^{q} \int_{|w|>R} B_{\left(|g|^{q}, \psi\right)}^{\frac{p}{p-q}}(w) d m(w) \rightarrow 0
$$


when $R \rightarrow \infty$. For such sufficiently big $R$, we estimate the remaining piece of integral as

$$
I_{n 1} \lesssim \sup _{|w| \leq R} \frac{\left|f_{n}^{\prime}(w)\right|^{q}}{(1+|w|)^{q}} \int_{|w| \leq R} e^{-\frac{q \alpha}{2}|w|^{2}} B_{\left(|g|^{q}, \psi\right)}(w) d m(w) \lesssim \sup _{|w| \leq R}\left|f_{n}(w)\right|^{q} \rightarrow 0
$$

as $n \rightarrow \infty$.

The proof of part (ii) of the theorem is very similar to the proof made above for part (i) and we omit it.

4.3. Proof of Corollary 2.1. (i) As pointed out earlier, for the special case when $\psi(z)=$ $z$, the operators $J_{(g, \psi)}$ reduce to the Volterra companion operator $J_{g}$. We may first assume that $g$ is a constant function and $q<\infty$. Then setting $\psi(z)=z$ in Theorem 2.1, we see

$$
\begin{array}{r}
\sup _{w \in \mathbb{C}} B_{\left(|g|^{q}, \psi\right)}(w) \simeq \sup _{w \in \mathbb{C}} \int_{\mathbb{C}}(|w|+1)^{q}\left|k_{w}(z)\right|^{q} \frac{e^{-\frac{q \alpha}{2}|z|^{2}}}{(1+|z|)^{q}} d m(z) \\
=\sup _{w \in \mathbb{C}} \int_{\mathbb{C}}(|w|+1)^{q} \frac{e^{-\frac{q \alpha}{2}|w-z|^{2}}}{(1+|z|)^{q}} d m(z)
\end{array}
$$

is finite. In a similar way, if $q=\infty$, then $M_{(g, \psi)}(z) \simeq 1$ for all points $z$ in $\mathbb{C}$. On the other hand, if $B_{\left(|g|^{q}, \psi\right)}$ is bounded, then by subharmonicity, we have

$$
\begin{array}{r}
\sup _{w \in \mathbb{C}} B_{\left(|g|^{q}, \psi\right)}(w) \geq \int_{D(w, 1)}(|w|+1)^{q}\left|k_{w}(z)\right|^{q} \frac{e^{-\frac{q \alpha}{2}|z|^{2}}|g(z)|^{q}}{(1+|z|)^{q}} d m(z) \\
\gtrsim \frac{|w g(w)|^{q}}{(1+|w|)^{q}},
\end{array}
$$

where we used the fact that $1+|z| \simeq 1+|w|$ whenever $z$ belongs to the disc $D(w, 1)$. The above estimate implies that

$$
\sup _{w \in \mathbb{C}}|g(w)|<\infty .
$$

Since $g$ is an entire function, this holds only when $g$ is a constant function. For $q=\infty$, the necessity is rather immediate because

$$
\infty>\sup _{z \in \mathbb{C}} M_{(g, \psi)}(z) \gtrsim \sup _{z \in \mathbb{C}}|g(z)| .
$$

We next show the claim that $M_{g}$ is bounded if and only if $g$ is a constant function again. Assuming that $M_{g}$ is bounded and $q<\infty$, we have

$$
\begin{aligned}
\left\|M_{g} k_{w}\right\|_{q}^{q} & =\frac{\alpha q}{2 \pi} \int_{\mathbb{C}}|g(z)|^{q}\left|k_{w}(z)\right|^{q} e^{-\frac{q \alpha}{2}|z|^{2}} d m(z) \\
& \gtrsim \int_{D(w, 1)}|g(z)|^{q} e^{-\frac{q \alpha}{2}|z-w|^{2}} d m(z) \geq|g(w)|^{q}
\end{aligned}
$$


for all $w$, where the last inequality follows by subharmonicity again. From this we deduce that $g$ is a constant function.

Conversely, assume that $g$ is a constant function. Then for each $f$ in $\mathcal{F}_{\alpha}^{p}$, we have $\left\|M_{g} f\right\|_{q} \simeq\|f\|_{q} \leq\|f\|_{p}$ where we use the inclusion property $\mathcal{F}_{\alpha}^{p} \subset \mathcal{F}_{\alpha}^{q}$ whenever $p \leq q$. For the case when $q=\infty$, observe that a constant $g$ implies $\left\|M_{g} f\right\|_{\infty} \simeq\|f\|_{\infty} \leq$ $\|f\|_{p}$ for any $p$ from which the boundedness of $M_{g}$ follows. On the other hand, if $M_{g}$ is bounded, then

$$
\left\|M_{g} k_{w}\right\|_{\infty} \geq\left|g(z) k_{w}(z)\right| e^{-\frac{\alpha}{2}|z|^{2}}
$$

for each $w$ and $z$ in $\mathbb{C}$. In particular when we set $w=z$, we find that $|g(w)|$ is uniformly bounded independent of $w$ from which the assertion follows.

To prove the corresponding statements for compactness, we may note that if $g=0$, then both $J_{g}$ and $M_{g}$ are the trivial zero maps and they are compact. On the other hand, suppose $J_{g}$ is compact. Then by Theorem 2.1 and (4.42), we have that

$$
0=\lim _{|w| \rightarrow \infty} B_{\left(|g|^{q}, \psi\right)}(w) \geq \lim _{|w| \rightarrow \infty}|g(w)| .
$$

This holds only if $g=0$. The case for $q=\infty$ is straightforward.

Since $k_{w}$ is a unit norm functions which converges uniformly to zero on compact subset of $\mathbb{C}$ as $|w| \rightarrow \infty$, by (4.43) we have

$$
0=\lim _{|w| \rightarrow \infty}\left\|M_{g} k_{w}\right\|_{q} \geq \lim _{|w| \rightarrow \infty}|g(w)|
$$

from which the desired conclusion follows again.

(ii) Since the sufficiency is trivial, we shall assume that $J_{g}$ is bounded (compact) and proceed to show that $g$ is the zero function. We may first suppose $p<\infty$. Then an application of part (i) of Theorem 2.2 and subharmonicity give

$$
\begin{aligned}
\int_{\mathbb{C}} B_{\left(|g|^{q}, \psi\right)}^{\frac{p}{p-q}}(w) d m(w) & \geq \int_{\mathbb{C}}\left(\int_{D(w, 1)} \frac{(|w|+1)^{q}\left|k_{w}(z) g(z)\right|^{q}}{(1+|z|)^{q} e^{\frac{\alpha q}{2}|z|^{2}}} d m(z)\right)^{\frac{p}{p-q}} d m(w) \\
& \geq \int_{\mathbb{C}}|g(w)|^{\frac{q p}{p-q}} d m(w) .
\end{aligned}
$$

This holds only if $g=0$. On the other hand, if $p=\infty$, then we repeat the above algorithm with exponent $p /(p-q)$ replaced by 1 and to easily arrive at the same conclusion.

To prove that bounded (compact) $M_{g}$ implies $g$ is the zero function, we act as in the proof of the necessity of the condition in Theorem 2.2. We in particular follow the rout leading to the estimate (4.35). In this case, the corresponding estimate would be

$$
\int_{\mathbb{C}}\left(\sum_{j=1}^{\infty}\left|c_{j}\right|^{2}\left|k_{z_{j}}(z)\right|^{2}\right)^{\frac{q}{2}} d \mu(z) \lesssim\left\|M_{g}\right\|^{q}\left\|\left(c_{j}\right)\right\|_{\ell^{p}}^{q}
$$


where $d \mu_{g}(z)=|g(z)|^{q} d m(z)$. On the other hand,

$$
\begin{array}{r}
\int_{\mathbb{C}}\left(\sum_{j=1}^{\infty}\left|c_{j}\right|^{2}\left|k_{z_{j}}(z)\right|^{2}\right)^{\frac{q}{2}} d \mu_{g}(z) \geq \frac{1}{N_{\max }} \sum_{j=1}^{\infty} \int_{D\left(z_{j}, 2 r\right)}\left|c_{j} k_{z_{j}}(z) g(z)\right|^{q} e^{-\frac{\alpha q}{2}|z|^{2}} d m(z) \\
\gtrsim \sum_{j=1}^{\infty} \int_{D\left(z_{j}, 2 r\right)}\left|c_{j}\right|^{q}|g(z)|^{q} d m(z),
\end{array}
$$

where the first inequality follows by Lemma 3.4. Since the sequence $\left(c_{j}\right)_{j \in \mathbb{N}}$ was chosen arbitrarily from $\ell^{p}$, the above relation together with (4.45) implies the sequence

$$
\int_{D\left(z_{j}, 2 r\right)}|g(z)|^{q} d m(z)
$$

belongs to $l^{p /(p-q)}$ for $p<\infty$ and $l^{1}$ for $p=\infty$. By the subharmonicity, we have

$$
|g(w)|^{q} \lesssim \int_{D\left(z_{j}, 2 r\right)}|g(z)|^{q} d m(z)
$$

for each $w$ in the disc $D\left(z_{j}, 3 r / 2\right)$ and for each $i \geq 1$. From this it follows for finite $p$ that

$$
\begin{aligned}
\int_{\mathbb{C}}|g(w)|^{\frac{p q}{p-q}} d m(w) \lesssim \sum_{j=1}^{\infty} & \left(\int_{D\left(z_{j}, 3 r / 2\right)}|g(z)|^{q} d m(z)\right)^{\frac{p}{p-q}} \\
& \lesssim \sum_{j=1}^{\infty}\left(\int_{D\left(z_{j}, r\right)}|g(z)|^{q} d m(z)\right)^{\frac{p}{p-q}}<\infty .
\end{aligned}
$$

Seemingly, if $p=\infty$, then (4.46) ensures

$$
\int_{\mathbb{C}}|g(w)|^{q} d m(w) \lesssim \sum_{j=1}^{\infty} \int_{D\left(z_{j}, r\right)}|g(z)|^{q} d m(z)<\infty .
$$

Since $g$ is analytic, the estimates in (4.47) and (4.48) hold only if $g$ is the zero function as asserted. Interested readers may consult [17] to see why the zero function is the only $L^{q}$ integrable entire function on $\mathbb{C}$.

\section{REFERENCES}

[1] A. Aleman, A class of integral operators on spaces of analytic functions, Topics in complex analysis and aperator theory, 3-30, Univ. Málaga, Málaga, 2007.

[2] A. Aleman and J. Cima, An integral operator on $H^{p}$ and Hardy's inequality, J. Anal. Math. 85 (2001), 157-176.

[3] A. Aleman and A. Siskakis, An integral operator on $H^{p}$, Complex Variables 28 (1995), $149-158$. 
[4] A. Aleman and A. Siskakis, Integration operators on Bergman spaces, Indiana University Math J. 46 (1997), 337-356.

[5] O. Constantin, Volterra type integration operators on Fock spaces, Proc. Amer. Math. Soc. 140 (12) (2012), 4247-4257.

[6] R. Fleming and J. Jamison, Isometries on Banach spaces, function spaces, monographs and surveys in pure and applied mathematics, vol. 129. Chapman and Hall/CRC, Boca Raton (2003).

[7] Z. Hu and X. Lv, Toeplitz operators from one Fock space to another, Integr. Equ. Oper. Theory 70 (2011), 541-559.

[8] J. Isralowitz and K. Zhu, Toeplitz operators on the Fock space, Integr. Equ. Oper. Theory, 66 (2010), no. 4, 593-611.

[9] S. Janson, J. Peetre, and R. Rochberg, Hankel forms and the Fock space, Rev. Mat. Iberoamericana 3 (1987), 61-138.

[10] S. Li, Volterra composition operators between weighted Bergman spaces and Bloch type spaces, J. Korean Math. Soc. 45 (2008), 229-248.

[11] S. Li and S. Stević, Generalized composition operators on Zygmund spaces and Bloch type spaces, J. Math. Anal. Appl. 338 (2008), 1282-1295.

[12] S. Li and S. Stević, Products of Volterra type operator and composition operator from $H^{\infty}$ and Bloch spaces to the Zygmund space, J. Math. Anal. Appl. 345 (2008), 40-52.

[13] D. Luecking, Embedding theorems for space of analytic functions via Khinchine's inequality, Michigan Math. J. 40 (1993), 333-358.

[14] T. Mengestie, Product of Volterra type integral and composition operators on weighted Fock spaces, J. Geom. Anal., 24(2014), 740-755.

[15] T. Mengestie, Volterra type and weighted composition operators on weighted Fock spaces, Integr. Equ. Oper. Theory, 76 (2013), no 1, 81-94.

[16] C. Pommerenke, Schlichte Funktionen und analytische Funktionen von beschránkter mittlerer Oszillation, Commentarii Mathematici Helvetici 52 (1977), no. 4, 591-602.

[17] Rashkovskii, A. Rashkovskii, Classical and new loglog-theorems, Expo. Math., 27 (2009), no. 4, 271-287.

[18] K. Seip and El. Youssfi, Hankel operators on Fock spaces and related Bergman kernel estimates, Journal of Geometric Anal., 23(2013), 170-201.

[19] A. Siskakis, Volterra operators on spaces of analytic functions-a survey, Proceedings of the first advanced course in operator theory and complex analysis, 51-68, Univ. Sevilla Secr., Seville, 2006.

[20] S. Stević, Weighted composition operators between Fock-type spaces in $\mathbb{C}^{N}$, Appl. Math. Comput. 215 (2009), 2750-2760.

[21] S. I. Ueki, Weighted composition operator on some function spaces of entire functions, Bull. Belg. Math. So. Simon Stevin 17 (2010), 343-353.

[22] S. I. Ueki, On the Li-Stević integral type operators from weighted Bergman spaces into $\alpha$-Zygmund spaces, Integral Equations Operator Theory 74 (1) (2012), 137-150.

[23] R. Wallstén, The $S^{p}$ Criterion for Hankel forms on the Fock space, $0<p<1$, Math. Scand. 64 (1989), 123-132.

[24] K. Zhu, Spaces of Holomorphic Functions in the Unit Ball, Springer-Verlag, New York, 2005. 
Department of Mathematical Sciences, Stord/Haugesund University College (HSH), KLINGENBERGVEGEN 8, N-5414 STORD, NORWAY

E-mail address: tesfantnu@gmail.com 